\title{
Inverse Problem for Shape Reconstruction of Plate-Thinning by Guided SH-Waves
}

\author{
Bin Wang ${ }^{1, * 1}$ and Sohichi Hirose $\mathrm{e}^{2, * 2}$ \\ ${ }^{1}$ Department of Civil and Environmental Engineering, Tokyo Institute of Technology, Tokyo 152-8552, Japan \\ ${ }^{2}$ Department of Mechanical and Environmental Informatics, Tokyo Institute of Technology, Tokyo 152-8552, Japan
}

In this paper, a new method for shape reconstruction of local plate thinning from reflection coefficients of guided SH-waves, based on guided wave scattering theory, is presented. The Green's function for the SH-wave problem is used to express the reflected wave field in an integral form in terms of the surface shape of flaw and the total wave displacement. By introducing the Born approximation and the far-field approximation into the integral form of the reflected wave, the depth of plate thinning is obtained as a function of the horizontal coordinate by performing the inverse Fourier transform of the reflection coefficients at various frequencies. Numerical examples are given and the accuracy of proposed inverse approach is discussed by means of parametric comparisons for different wave modes, frequency ranges and various thinning shapes. [doi:10.2320/matertrans.I-M2012823]

(Received November 30, 2011; Accepted July 10, 2012; Published August 29, 2012)

Keywords: guided SH-wave, plate thinning reconstruction, inverse problem, Born approximation, Fourier transform

\section{Introduction}

Guided wave propagation in elastic waveguides has been investigated by many researchers, since guided waves are considered to be a potential useful tool for non-destructive inspection and health monitoring, ${ }^{1-3)}$ especially in case of long-range inspections. Various methods have been suggested for applications of guided waves in NDI.

The traditional but frequently used approaches include the pulse-echo, pitch-catch and array-based methods. Although they were developed many years ago, their improved applications with new techniques and algorithms are still proposed by researchers. Wilcox ${ }^{4)}$ and Rajagopalan et al. ${ }^{5)}$ developed "phased addition algorithms" and used an omnidirectional transducer and receiver array system for Lamb wave modes to inspect over a large span of the plate for defect locations and directivities. While Nishino et al. ${ }^{6}$ introduced an efficient transduction method to enhance the echo signals of the first torsional mode when inspecting defects in pipes.

Tomographic technologies using guided waves have also been well studied. Jansen and Hutchins ${ }^{7,8)}$ made the pioneer works concerning Lamb wave tomography and suggested the filter back-projection method. Malyarenko and Hinders ${ }^{9)}$ and Leonard et al. ${ }^{10)}$ developed an iterative reconstruction procedure based on curved ray algebraic reconstruction technique (ART). For industrial applications, Gao et al. ${ }^{11)}$ and Hay et al. $^{12)}$ applied the back-projection and ART methods to aircraft wing inspection. However, due to mode conversion and dispersion, the guided waves tend to follow more complicated propagation rules, which may make conventional forms of bulk wave tomography invalid in some cases. ${ }^{9)}$

The methods mentioned above only make use of arrival time of reflected/scattered wave signals, thus they tend to detect the locations instead of shapes of defects. Some

\footnotetext{
${ }^{* 1}$ Graduate Student, Tokyo Institute of Technology

${ }^{* 2}$ Corresponding author, E-mail: shirose@cv.titech.ac.jp
}

researchers have been trying various inverse approaches making use of more detailed data of scattered wave fields. Liu and Lam $^{13)}$ firstly applied the frequency domain strip element method (SEM) in the forward analysis of the sandwich plate in which guided $\mathrm{SH}$-waves are scattered by rectangular flaws. Using the approach, Wang et al. ${ }^{14)}$ studied the effect of different flaw length on the wave field's displacement distribution patterns, and defined the "damage factor" of the flaw identifying its extent of detriment to the sandwich plate. Liu and $\mathrm{Chen}^{15)}$ and $\mathrm{Wu}$ et al. ${ }^{16)}$ tried to adopt genetic algorithm (GA) to detect the presence and size of a flaw in a plate-like waveguide, using time-harmonic response obtained from SEM as input data. However, the "damage factor" is an abstract terminology, and the adoption of GA requires a lot of training and may introduce uncertainty. Meanwhile, Rose and Wang studied the possibility of inverse analysis in a Mindlin plate. They deduced a dyadic Green's function for a point moment or force, ${ }^{17,18)}$ by the help of which they derived the far-field radiation pattern due to piezoelectric radiators. Applying the Green's function and the Born approximation, they showed a relationship between far-field scattering amplitude and spatial Fourier transform of local homogeneity, based on which an approach to reconstruct weak flexural inhomogeneity is proposed. ${ }^{19)}$ However, the Mindlin plate model is valid only if the wavelength is comparable or longer than plate thickness, which restricts the choice of frequency range for guided wave. ${ }^{17)}$

In this paper, a new inverse analysis approach to reconstruct plate-thinning shapes based on scattering theory of time-harmonic guided SH-waves is proposed. Firstly, an artificial thinning flaw is introduced on the surface of a 2-D plate model, and the reflection coefficients at different frequencies for each guided $\mathrm{SH}$-wave mode are calculated through a forward analysis. Meanwhile, the Green's function in a traction-free plate waveguide for $\mathrm{SH}$-wave is deduced, with the help of which the reflection wave field is expressed in the form on the boundary of local inhomogeneous area. After that, by adopting the Born 
approximation and the far-field approximation to the flaw area, the flaw shape function is obtained by performing the inverse Fourier transform to the expression of the reflection coefficients.

In Section 2, the forward and inverse problems are stated. Section 3 introduces Green's function for SH-wave problem in a plate. Numerical examples are illustrated in Section 4 to show the method's validity, and proper choices of wave modes and frequency range are discussed.

\section{Basic Characteristics of Guided SH-Waves and Problem Definition}

\subsection{Guided SH-wave modes and mode orthogonality}

It is assumed that the particles in a plate vibrate in the anti-plane direction, say, $x_{3}$-direction, and the vertical axis pointing downward is termed $x_{2}$, as shown in Fig. 1. The equation of motion for the $\mathrm{SH}$-wave concerning displacement field $u$ can be expressed as follows.

$$
\left(\frac{\partial^{2}}{\partial x_{1}^{2}}+\frac{\partial^{2}}{\partial x_{2}^{2}}\right) u-\frac{1}{V_{\mathrm{S}}^{2}} \frac{\partial^{2} u}{\partial t^{2}}=0
$$

Here $V_{\mathrm{S}}$ is the $\mathrm{SH}$-wave velocity defined as $V_{S}=\sqrt{\mu / \rho}$, where $\mu$ is the shear stiffness and $\rho$ is the density of a plate. For a time-harmonic SH-wave, the displacement has the form of $u=\tilde{u}\left(x_{1}, x_{2}\right) e^{+i \omega t}$. When substituted into eq. (1a) and the traction-free boundary conditions for an intact plate:

$$
\tau_{23}=\mu \partial u / \partial x_{2}=0 \text { at } x_{2}= \pm b
$$

where $2 b$ is the plate depth, the displacement field for the $n$th guided SH-wave mode $(n=0,1,2, \ldots)$ is obtained in the form of

$$
\tilde{u}_{n}=A_{n} f_{n}\left(\beta_{n} x_{2}\right) e^{ \pm i \xi_{n} x_{1}}
$$

where $\beta_{n}=n \pi / 2 b, \quad \xi_{n}=\sqrt{\omega^{2} / V_{s}^{2}-\beta_{n}^{2}}$, and $A_{n}$ is the amplitude coefficient. In eq. (2a), $f_{n}(x)$ is a function defined as:

$$
f_{n}(x)= \begin{cases}\cos x & \text { for } n=0,2,4, \ldots \\ & (\text { symmetric mode) } \\ \sin x & \text { for } n=1,3,5, \ldots \\ & \text { (antisymmetric mode) }\end{cases}
$$

It has been proved by Auld ${ }^{20)}$ that different propagating guided wave modes are orthogonal with each other. When a body force is absent, for two guided SH-wave modes, say $\left(\tilde{u}_{i}, \tilde{\tau}_{i}\right)$ and $\left(\tilde{u}_{j}, \tilde{\tau}_{j}\right)$, in the waveguide, we have the following relation:

$$
\left(\xi_{j}^{*}-\xi_{i}\right) \int_{-b}^{+b}\left(\tilde{u}_{i} \tilde{\tau}_{j}^{*}-\tilde{u}_{j}^{*} \tilde{\tau}_{i}\right) d x_{2}=0
$$

where $\tilde{\tau}_{i}$ is the shear stress $\tilde{\tau}_{13}$ of the $i$ th mode, asterisks stand for complex conjugates, and tildes mean the wave fields without time factor of $e^{+i \omega t}$.

In an actual plate waveguide, the total wave fields $\tilde{u}^{\text {tot }}$ and $\tilde{\tau}^{\text {tot }}$ are comprised of a series of wave modes as:

$$
\tilde{u}^{\mathrm{tot}}=\sum_{i} A_{i} \tilde{u}_{i}, \quad \tilde{\tau}^{\mathrm{tot}}=\sum_{i} A_{i} \tilde{\tau}_{i} .
$$

If the total wave fields are obtained numerically, the coefficient $A_{i}$ for the $i$ th mode included in the total wave fields can be determined using the mode orthogonality as

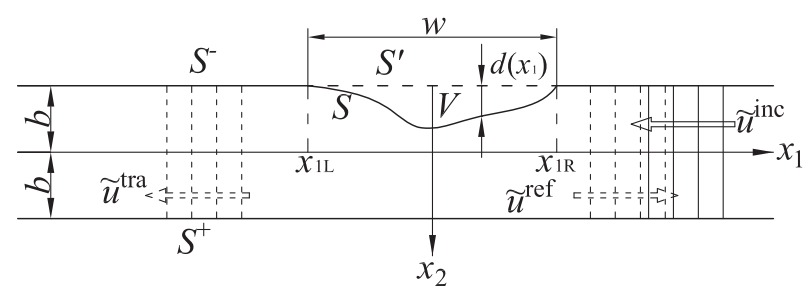

Fig. 1 Reflection and transmission of an incident guided SH-wave by a plate thinning.

eq. (5), which helps us separating modes from the wave field in forward analysis.

$$
A_{i}=\frac{\int_{-b}^{b}\left(\tilde{u}^{\mathrm{tot}} \tilde{\tau}_{i}^{*}-\tilde{u}_{i}^{*} \tilde{\tau}^{\mathrm{tot}}\right) d x_{2}}{\int_{-b}^{b}\left(\tilde{u}_{i} \tilde{\tau}_{i}^{*}-\tilde{u}_{i}^{*} \tilde{\tau}_{i}\right) d x_{2}}
$$

\subsection{Forward and inverse problems}

As shown in Fig. 1, a thinning part with the width $w$ and the depth $d$ is located on the top surface of the plate. It is assumed that the depth $d$ is much smaller than the plate thickness $2 b$, i.e., $d \ll b$. The original top and bottom surfaces are termed as $S^{-}$and $S^{+}$, while the surface of thinning part as $S$ and the original surface between horizontal coordinates $x_{1 \mathrm{~L}}$ and $x_{1 \mathrm{R}}$ as $S^{\prime}$. The volume bounded by $S$ and $S^{\prime}$ is $V$. It is assumed that the incident wave is the guided $\mathrm{SH}$-wave with the $n$th mode, propagating from the right side to left and being reflected back by the thinning part, and the reflected wave with the same mode as the incident wave mode is observed at far field.

According to eq. (2), the displacements for the incident and reflected guided SH-waves, $\tilde{u}^{\text {inc }}$ and $\tilde{u}^{\text {ref }}$, can be expressed as:

$$
\tilde{u}^{\text {inc }}=A_{n}^{\text {inc }} f_{n}\left(\beta_{n} x_{2}\right) e^{+i \xi_{n} x_{1}}, \quad \tilde{u}^{\text {ref }}=A_{n}^{\text {ref }} f_{n}\left(\beta_{n} x_{2}\right) e^{-i \xi_{n} x_{1}} .
$$

The forward problem is to determine the amplitude $A_{n}^{\text {ref }}$ of reflected waves due to the surface thinning subjected to a single-mode incident wave with the given amplitude $A_{n}^{\text {inc }}$. We use the mode exciting method ${ }^{21)}$ to obtain the amplitudes $A_{n}^{\text {ref }}$ of reflected waves as a function of wavenumber $\xi_{n}$, or, the frequency $\omega$. In this method, a truncated waveguide with enough length is chosen as a model for calculation, in which a thinning area is located right at the middle of the model. By exerting a proper displacement set on both the truncated ends, the wave field within the truncated waveguide is obtained by finite element method, and eq. (5) is applied to decompose the wave field into a series of orthogonal wave modes. By exerting different sets of displacement conditions at artificial boundaries, the amplitudes of reflected waves for a particular incident wave mode can be determined by solving a linear equation set with respect to the amplitudes of various wave modes propagating back and forth in the truncated waveguide.

As explained in the next section, the inverse problem is to obtain the function $d\left(x_{1}\right)$, representing the shape of plate thinning, from the reflection coefficient, defined by $C^{\text {ref }}=A_{n}^{\text {ref }} / A_{n}^{\text {inc }}$, which has been obtained as a function of frequency. 


\section{The Inverse Analysis}

\subsection{Green's function}

In order to construct an integral expression for a reflected wave, we derive the Green's function, $\tilde{U}(\boldsymbol{x}, \boldsymbol{X})$, which represents the anti-plane displacement at the field point $\boldsymbol{x}=\left(x_{1}, x_{2}\right)$ due to a time harmonic point force exerted at the source point $\boldsymbol{X}=\left(X_{1}, X_{2}\right)$ in an intact plate.

The Green's function $\tilde{U}(\boldsymbol{x}, \boldsymbol{X})$ satisfies the equation of motion:

$$
\nabla^{2} \tilde{U}(\boldsymbol{x}, \boldsymbol{X})+k^{2} \tilde{U}(\boldsymbol{x}, \boldsymbol{X})=-\delta(\boldsymbol{x}-\boldsymbol{X}) / \mu
$$

and the traction free boundary condition:

$$
\tilde{T}(\boldsymbol{x}, \boldsymbol{X}) \equiv \mu \frac{\partial}{\partial n(\boldsymbol{x})} \tilde{U}(\boldsymbol{x}, \boldsymbol{X})=0 \text { on } x_{2}= \pm b
$$

where $k=\omega / V_{\mathrm{S}}$ is the shear wave number and $\partial / \partial n$ indicates the normal derivative.

It is assumed that the Green's function $\tilde{U}$ contains two parts; the fundamental solution $\tilde{U}^{\text {inc }}$, which is a particular solution of eq. (7), representing a cylindrical wave in an infinite domain due to a point force, and the additional term $\tilde{U}^{\text {ref }}$, which represents reflected waves when the cylindrical wave meets the top and bottom boundaries of a plate without defect. Finally, $\tilde{U}$ is expressed as

$$
\begin{aligned}
\tilde{U}(\boldsymbol{x}, \boldsymbol{X})= & \tilde{U}^{\mathrm{inc}}(\boldsymbol{x}, \boldsymbol{X})+\tilde{U}^{\mathrm{ref}}(\boldsymbol{x}, \boldsymbol{X}) \\
= & \frac{1}{4 \pi \mu} \int_{-\infty}^{\infty} \frac{e^{-R\left|x_{2}-X_{2}\right|}}{R} e^{-i \xi\left(x_{1}-X_{1}\right)} d \xi \\
& +\frac{1}{4 \pi \mu} \int_{-\infty}^{\infty}\left(A^{+} e^{-R x_{2}}+A^{-} e^{+R x_{2}}\right) e^{-i \xi\left(x_{1}-X_{1}\right)} d \xi
\end{aligned}
$$

where $R=\sqrt{\xi^{2}-k^{2}}(|\xi| \geq k), \quad i \sqrt{k^{2}-\xi^{2}}(|\xi| \leq k)$, while $A^{+}$and $A^{-}$represent the undetermined amplitudes of "reflected waves" from lower and upper surfaces, respectively. Substituting eq. (9) into eq. (8), the amplitudes $A^{+}$ and $A^{-}$are solved. Then it follows that:

$$
\begin{aligned}
\tilde{U}(\boldsymbol{x}, \boldsymbol{X})= & \frac{1}{4 \pi \mu} \int_{-\infty}^{\infty}\left[\frac{e^{-R\left|x_{2}-X_{2}\right|}}{R}+\frac{e^{-2 R b}}{2 R\left(1+e^{-2 R b}\right)}\right. \\
& \times\left(e^{-R X_{2}}-e^{+R X_{2}}\right)\left(e^{-R x_{2}}-e^{+R x_{2}}\right) \\
& +\frac{e^{-2 R b}}{2 R\left(1-e^{-2 R b}\right)}\left(e^{-R X_{2}}+e^{+R X_{2}}\right) \\
& \left.\times\left(e^{-R x_{2}}+e^{+R x_{2}}\right)\right] e^{-i \xi\left(x_{1}-X_{1}\right)} d \xi .
\end{aligned}
$$

For $\left|x_{1}\right| \gg\left|X_{1}\right|$, the incident wave part $\tilde{U}^{\text {inc }}$ decays with the order of $O\left(\left|x_{1}\right|^{-1 / 2}\right)$, and the remaining $\tilde{U}^{\text {ref }}$ is expressed as the sum of a series of propagating guided SH-waves with no decay, which can be evaluated applying the residue theorem in complex analysis to the second and third integrands in eq. (10). Consequently, the far-field expression for the Green's function is obtained as

$$
\begin{aligned}
\tilde{U}(\boldsymbol{x}, \boldsymbol{X}) \cong & \tilde{U}^{\mathrm{far}}(\boldsymbol{x}, \boldsymbol{X}) \\
= & -\frac{i}{4 b \mu \xi_{0}} e^{-i \xi_{0}\left|x_{1}-X_{1}\right|}-\sum_{j} \frac{i}{2 b \mu \xi_{j}} f_{j}\left(\beta_{j} x_{2}\right) \\
& \times f_{j}\left(\beta_{j} X_{2}\right) e^{-i \xi_{j}\left|x_{1}-X_{1}\right|} \text { as }\left|x_{1}\right| \gg\left|X_{1}\right|
\end{aligned}
$$

where the functions $f_{n}(x)$ are defined in eq. (2b).

\subsection{Inverse problem formulation}

The inverse problem formulation is based on the integral expression for the scattered wave by a plate-thinning area. With use of the Green's function $\tilde{U}(\boldsymbol{x}, \boldsymbol{X})$, the scattered wave can be written in the following form:

$$
\tilde{u}^{\mathrm{sca}}(\boldsymbol{x})=\int_{S}\left[\tilde{u}^{\mathrm{tot}}(\boldsymbol{X}) \tilde{T}(\boldsymbol{X}, \boldsymbol{x})-\mu \frac{\partial \tilde{u}^{\mathrm{tot}}(\boldsymbol{X})}{\partial n(\boldsymbol{X})} \tilde{U}(\boldsymbol{X}, \boldsymbol{x})\right] d s(\boldsymbol{X})
$$

where $\tilde{u}^{\text {sca }}$ and $\tilde{u}^{\text {tot }}$ represent scattered and total displacement fields, respectively. Considering the traction-free boundary condition of $\mu \partial \tilde{u}^{\text {tot }} / \partial n=0$ on the surface $S$, we obtain:

$$
\begin{aligned}
\tilde{u}^{\mathrm{sca}}(\boldsymbol{x}) & =\int_{S} \tilde{u}^{\mathrm{tot}}(\boldsymbol{X}) \tilde{T}(\boldsymbol{X}, \boldsymbol{x}) d s(\boldsymbol{X}) \\
& =\int_{S} \tilde{u}^{\mathrm{tot}}(\boldsymbol{X}) \mu \frac{\partial \tilde{U}(\boldsymbol{X}, \boldsymbol{x})}{\partial n(\boldsymbol{X})} d s(\boldsymbol{X}) .
\end{aligned}
$$

Since $d \ll b$, the scattering process due to plate thinning is supposed to be weak. Using the Born approximation, ${ }^{22,23)}$ therefore, the total displacement $\tilde{u}^{\text {tot }}$ in eq. (13) is approximated by the incident wave $\tilde{u}^{\text {inc }}$. Thus we have

$$
\tilde{u}^{\mathrm{sca}}(\boldsymbol{x}) \approx \int_{S} \tilde{u}^{\text {inc }}(\boldsymbol{X}) \mu \frac{\partial \tilde{U}(\boldsymbol{X}, \boldsymbol{x})}{\partial n(\boldsymbol{X})} d s(\boldsymbol{X})
$$

In light of Gauss's theorem and eq. (7), the surface integral in eq. (14) is converted into the integral over the volume $V$ bounded by $S$ and $S^{\prime}$ as follows.

$$
\begin{aligned}
\tilde{u}^{\text {sca }}(\boldsymbol{x}) \approx & \int_{V}\left[-k^{2} \tilde{u}^{\text {inc }}(\boldsymbol{X}) \mu \tilde{U}(\boldsymbol{X}, \boldsymbol{x})\right. \\
& \left.+\mu \frac{\partial \tilde{U}(\boldsymbol{X}, \boldsymbol{x})}{\partial X_{i}} \frac{\partial \tilde{u}^{\text {inc }}(\boldsymbol{X})}{\partial X_{i}}\right] d V(\boldsymbol{X})
\end{aligned}
$$

It is also assumed that the reflected waves of the same mode as the incident wave mode are received at far field, namely, $x_{1} \gg b>\left|X_{1}\right|$ in eq. (15). Then the far field approximation for the Green's function given by eq. (11) can be substituted into eq. (15). If both incident and reflected waves are the $n$th symmetric mode, for example, the following expressions are introduced into eq. (15):

$$
\begin{aligned}
\tilde{u}^{\text {inc }}(\boldsymbol{X}) & =A_{n}^{\mathrm{inc}} \cos \left(\beta_{n} X_{2}\right) e^{i \xi_{n} X_{1}} \\
\tilde{U}(\boldsymbol{X}, \boldsymbol{x}) & \approx \tilde{U}^{\mathrm{far}}(\boldsymbol{X}, \boldsymbol{x}) \\
& =-\frac{i}{2 b \mu \xi_{n}} \cos \left(\beta_{n} x_{2}\right) \cos \left(\beta_{n} X_{2}\right) e^{-i \xi_{n}\left(x_{1}-X_{1}\right)}
\end{aligned}
$$

where $n$ is a positive even number. Then the reflected wave can be expressed as follows:

$$
\begin{aligned}
\tilde{u}^{\mathrm{ref}}(\boldsymbol{x})= & \lim _{x_{1} \gg b} \tilde{u}^{\mathrm{sca}}(\boldsymbol{x}) \\
\cong & \frac{i}{2 b} A_{n}^{\mathrm{inc}} \int_{V} \frac{\xi_{n}^{2}+k^{2} \cos \left(2 \beta_{n} X_{2}\right)}{\xi_{n}} e^{2 i \xi_{n} X_{1}} d V(\boldsymbol{X}) \\
& \times \cos \left(\beta_{n} x_{2}\right) e^{-i \xi_{n} x_{1}} \\
\triangleq & A_{n}^{\mathrm{ref}} \cos \left(\beta_{n} x_{2}\right) e^{-i \xi_{n} x_{1}}
\end{aligned}
$$

where the integral multiplied by constants corresponds to the amplitude $A_{n}^{\text {ref }}$ of the reflected wave. Referred to Fig. 1, the volume integral can be viewed as multiple integrals with respect to $X_{1}$ and $X_{2}$ coordinates. The integration for $X_{2}$ ranges from $-b$ to $-b+d\left(X_{1}\right)$, which can be evaluated analytically, inducing the shallow flaw approximation of $d\left(X_{1}\right) \ll b$. Since $d\left(X_{1}\right)$ is non-zero only for $x_{1 \mathrm{~A}}<X_{1}<x_{1 \mathrm{~B}}$, 
the integral with respect to $X_{1}$ can be extended to the integral from $-\infty$ to $\infty$. It then follows that

$$
\begin{aligned}
C^{\mathrm{ref}} & =\frac{A_{n}^{\mathrm{ref}}}{A_{n}^{\text {inc }}} \\
& =\frac{i}{2 b} \frac{\xi_{n}^{2}+k^{2}}{\xi_{n}} \int_{x_{1 \mathrm{~A}}}^{x_{1 \mathrm{~B}}} d\left(X_{1}\right) e^{2 i \xi_{n} X_{1}} d X_{1} \\
& =\frac{i}{2 b} \frac{\xi_{n}^{2}+k^{2}}{\xi_{n}} \int_{-\infty}^{+\infty} d\left(X_{1}\right) e^{2 i \xi_{n} X_{1}} d X_{1} .
\end{aligned}
$$

The reflection coefficient $C^{\text {ref }}$, can be obtained as a function of $\xi_{n}$ by the mode-exciting method, and the integral on the RHS of eq. (18) is the Fourier transform of the shape function $d\left(X_{1}\right)$. Performing the inverse Fourier transform to (18), the plate-thinning shape function $d\left(X_{1}\right)$ is obtained as:

$$
d\left(X_{1}\right)=\frac{1}{2 \pi} \int_{-\infty}^{\infty} \frac{-2 i b \xi_{n}}{\xi_{n}^{2}+k^{2}} C^{\mathrm{ref}} e^{-2 i \xi_{n} X_{1}} d\left(2 \xi_{n}\right)
$$

Equation (19) is the basic equation to reconstruct the shape of plate-thinning from the reflection coefficients for the symmetric mode. The formulation for the anti-symmetric mode can be done similarly, and the final expression is the same as eq. (19).

\section{Numerical Examples}

In this section some numerical examples are illustrated to show the validity and effectiveness of the inverse reconstruction process for plate-thinning.

\subsection{Reflection coefficients}

As shown in Fig. 2, a sine-curved or V-shaped artificial thinning flaw is located at the top surface of a plate, with the width $w$ and the maximum depth $d_{\max }$. The incident wave is excited from the right side of the flaw. According to eq. (19), the reflection coefficients for the full- $\xi_{n}$ range are required to perform the integral on $(-\infty,+\infty)$. But in practice, an upper frequency limit $\Omega$ is defined and the reflection coefficients are calculated over the frequency range $\omega \in(0,+\Omega)$, which corresponds to the wavenumber $\xi_{n} \in\left(0,+\Xi_{n}\right)$, where $\Xi_{n}=\sqrt{\left(\Omega / V_{\mathrm{S}}\right)^{2}-(n \pi / 2 b)^{2}}$. Also, we have $C^{\text {ref }}\left(\xi_{n}\right)=C^{\text {ref* } *}\left(-\xi_{n}\right)$, where the asterisk symbol means the complex conjugate. So the integral range in eq. (19) can be taken as $\left(-\Xi_{n},+\Xi_{n}\right)$.

Figure 3 presents the absolute values of reflection coefficients for the first five modes, namely, 0th symmetric $(n=0), 1$ st anti-symmetric $(n=1), 1$ st symmetric $(n=2)$,

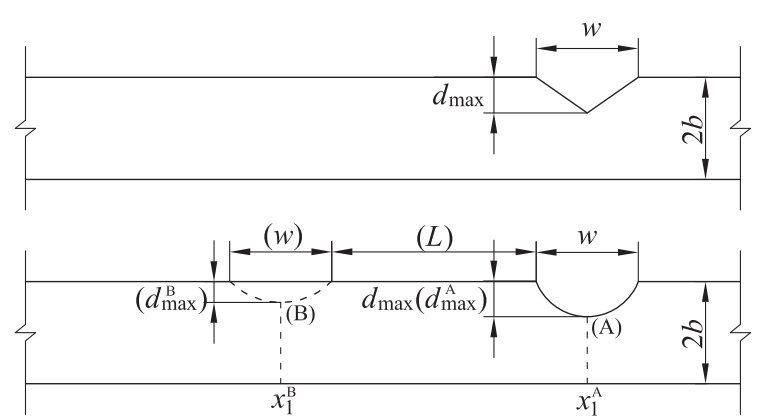

Fig. 2 Plate thinning types for numerical verifications.
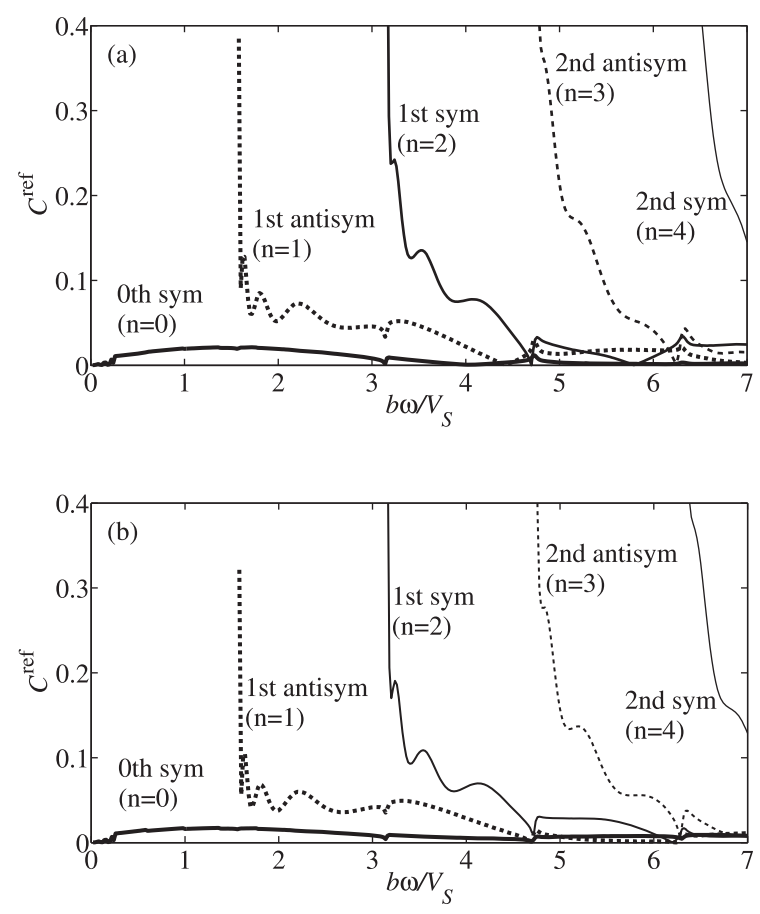

Fig. 3 Reflection coefficients of the first five SH-wave modes due to plate thinning. (a) Sine-curved flaw shape with $d_{\max } / b=0.05$ and $w / b=1.0$; (b) V-shaped flaw with $d_{\max } / b=0.05, w / b=1.0$.

2nd anti-symmetric $(n=3)$ and 2 nd symmetric $(n=4)$, when the scatterer is a sine-curve shaped (a) or V-shaped (b) thinning with $w / b=1.0$ and $d_{\max } / b=0.05$. Here the upper frequency limit is set to $\Omega=7.0 V_{S} / b$. It is seen that the reflection coefficient for each wave mode approaches a very large value near its cut-off frequency except for the 0th symmetric mode. These reflection coefficients are used as input data in the following inverse analysis.

\subsection{Effect of mode and flaw depth}

Figure 4 presents the comparison between the actual flaw shapes and the reconstructed results for two types of sinecurved and V-shaped flaws with the width $w / b=1$ and different maximum depths of $d_{\max } / b=0.05,0.15$ and 0.25 using the first three guided SH-wave modes with $n=0,1$ and 2. We can see the locations and basic features of flaws are found in all the cases. It is also observed that the right side of flaw, which is "illuminated" by the incident wave, is reconstructed better than the left side.

For quantitative discussion on reconstruction accuracy of the reconstructed results, we employ the mean square of error (MSE) defined as:

$$
\mathrm{MSE}=\sqrt{\frac{\sum_{i=1}^{N}\left(d_{i}-d_{i}^{0}\right)^{2}}{N}}
$$

where $N$ is the number of reconstructed points along the $x_{1}$ axis within the flaw and nearby zone of $-2 \leq x_{1} / b \leq 2 . d_{i}$ represents the reconstructed depth of flaw at the $i$ th point while $d_{i}^{0}$ is the actual depth at this point. The MSE values normalized by half-thickness $b$ for each case using different modes are shown in Table 1. 

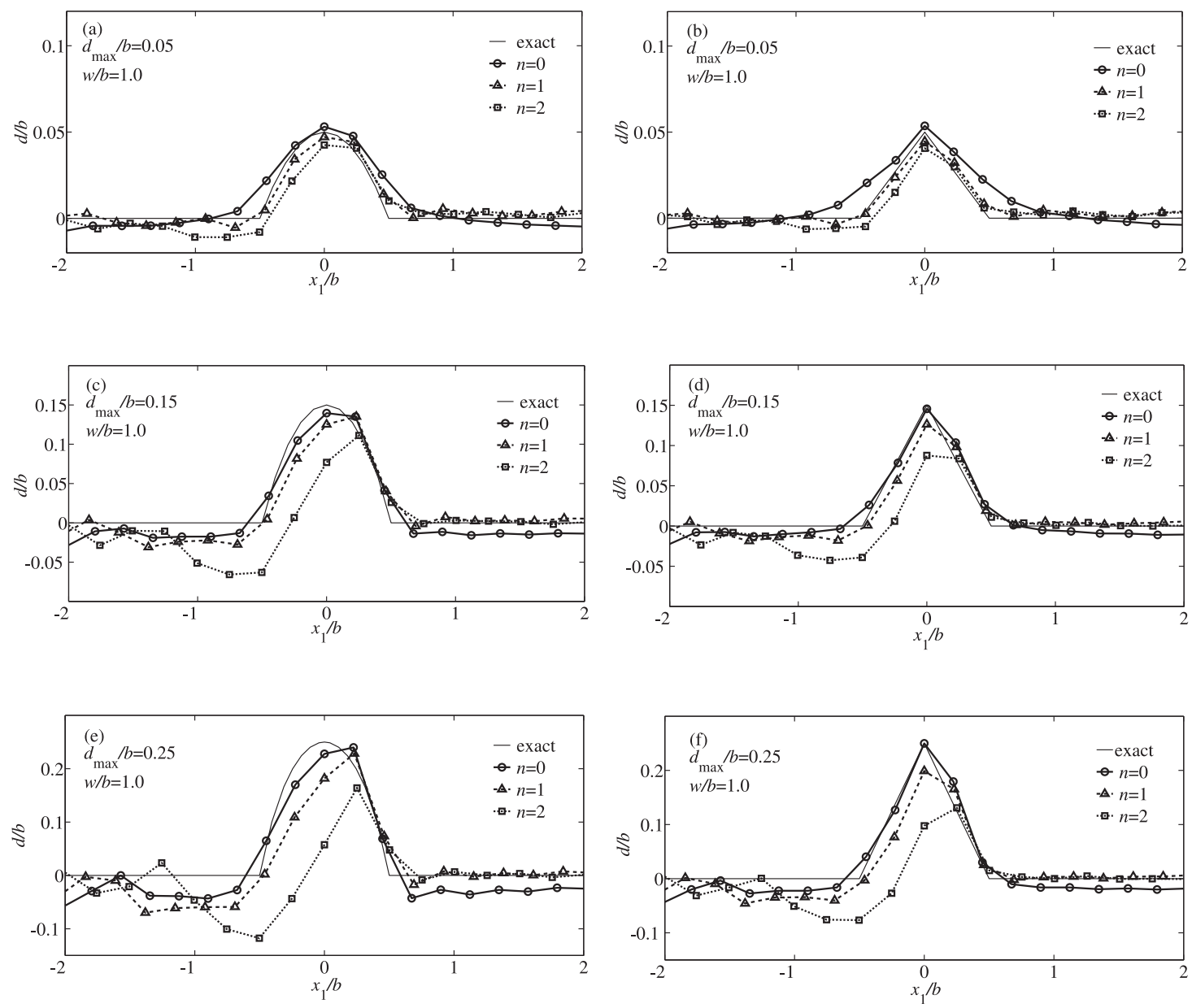

Fig. 4 Reconstructed shapes for flaws with various depths and modes. Left column: sine-curved flaw; Right column: V-shaped flaw.

Table 1 MSE of reconstructed flaw shapes with various values of $d_{\max }$.

\begin{tabular}{cccccc}
\hline Shape & $w / b$ & $d_{\max } / b$ & Mode $n=0$ & Mode $n=1$ & Mode $n=2$ \\
\hline Sine-shaped & \multirow{2}{*}{1.0} & 0.05 & 0.0060 & 0.0037 & 0.0074 \\
\cline { 3 - 6 } & & 0.15 & 0.014 & 0.019 & 0.044 \\
\cline { 3 - 6 } & 0.25 & 0.030 & 0.044 & 0.092 \\
\hline \multirow{2}{*}{ V-shaped } & \multirow{2}{*}{1.0} & 0.05 & 0.0074 & 0.0031 & 0.0052 \\
\cline { 3 - 5 } & & 0.15 & 0.0095 & 0.013 & 0.031 \\
\cline { 2 - 5 } & & 0.25 & 0.019 & 0.028 & 0.064 \\
\hline
\end{tabular}

From Table 1 it is seen that for any combination of flaw shape and wave mode, the values of MSE increase as the depth of flaw becomes larger, which means that our inverse method has better accuracy for shallower flaws. It is also found that the MSE values for the flaws with $d_{\max } / b=0.15$ and 0.25 become larger for higher modes, while the MSE values for the flaws with $d_{\max } / b=0.05$ are all very small and have no significant difference between all the three modes.

Table 1 also shows that in the case of $d_{\max } / b<0.25$ and $w / b=1$, the error of reconstructed flaw shapes does not exceed approximately $10 \%$ of the maximum depth.

\subsection{Effect of flaw width}

Figure 5 shows the comparison between results for $w / b=1.0$ and $w / b=2.0$ to illustrate how the flaw width
Table 2 MSE of reconstructed flaw shapes with various values of $w$.

\begin{tabular}{cccccc}
\hline Shape & $d_{\max } / b$ & $w / b$ & Mode $n=0$ & Mode $n=1$ & Mode $n=2$ \\
\hline Sine-shaped & 0.15 & 1.0 & 0.014 & 0.019 & 0.044 \\
\cline { 3 - 6 } & & 2.0 & 0.024 & 0.051 & 0.099 \\
\hline \multirow{2}{*}{ V-shaped } & 0.15 & 1.0 & 0.0095 & 0.013 & 0.031 \\
\cline { 3 - 6 } & & 2.0 & 0.015 & 0.032 & 0.071 \\
\hline
\end{tabular}

affects the reconstruction accuracy, while the $d_{\max } / b$ is kept at 0.15 in all the cases. The MSE values of reconstruction results are shown by Table 2 :

As seen in Table 2, the fundamental mode of $n=0$ shows the best accuracy among the results obtained by three wave modes. It is also observed that all MSE values for $w / b=2$ are larger than those for $w / b=1$. As seen in Figs. 5(b) and $5(\mathrm{~d})$, especially the shadow zones of longer flaws with $w / b=2$ are reconstructed worse using higher wave modes.

\subsection{Effect of frequency range}

Firstly, the effect of high-frequency range on inversion results is discussed. Figure 6(a) compares the reconstructed shapes for the sine-curved flaw using frequency ranges of $b \omega / V_{\mathrm{s}}=0-7.0 ; 0-5.0$ and $0-3.0$, respectively, using the fundamental 0 th symmetric mode $(n=0)$. Here $w / b=1.0$ and $d_{\max } / b=0.15$ are kept fixed. It is obvious that if the higher frequency range is removed from analysis region, the 

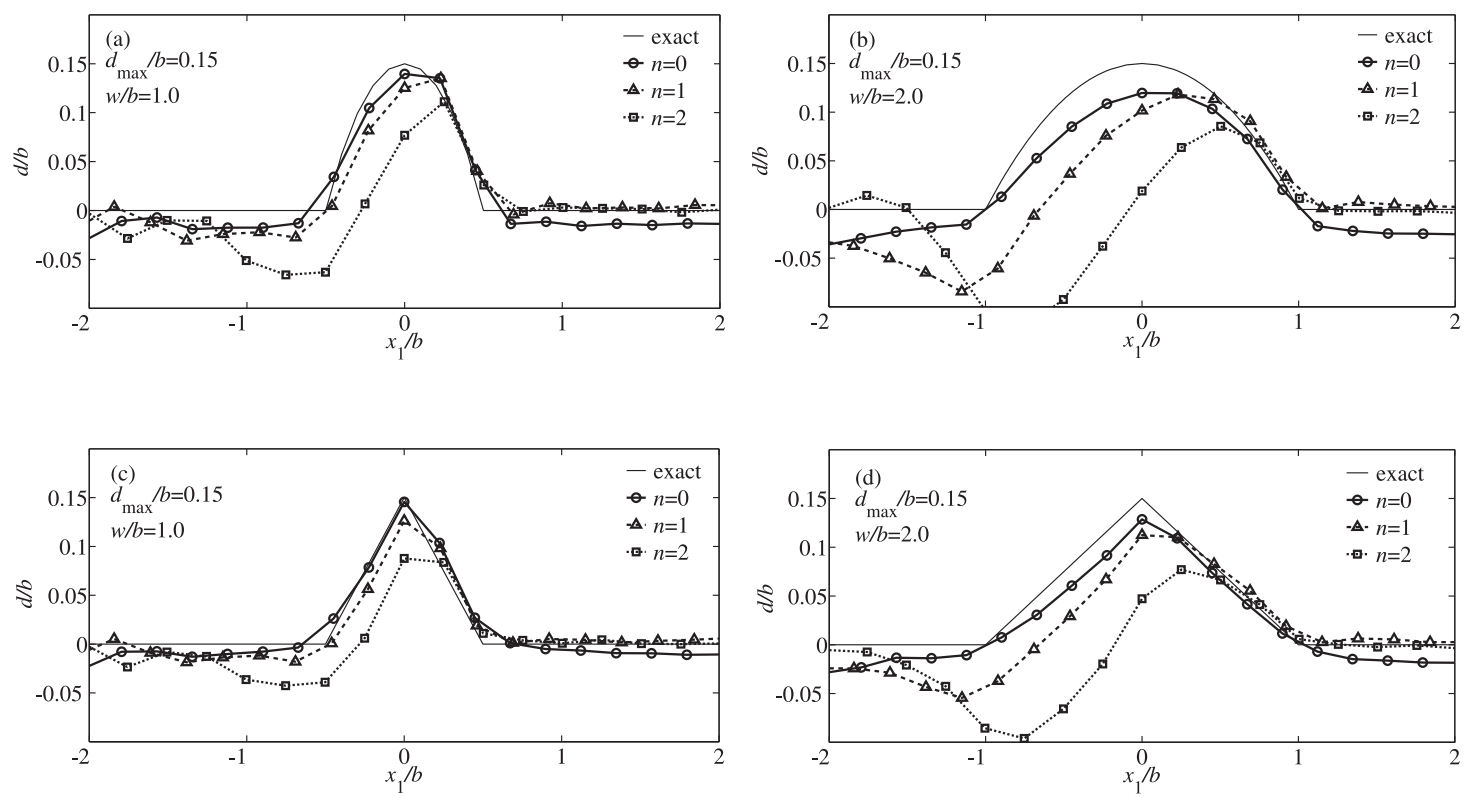

Fig. 5 Reconstructed shapes for flaws with different widths and modes. (a) and (b): Sine-curved flaw; (c) and (d): V-shaped flaw.
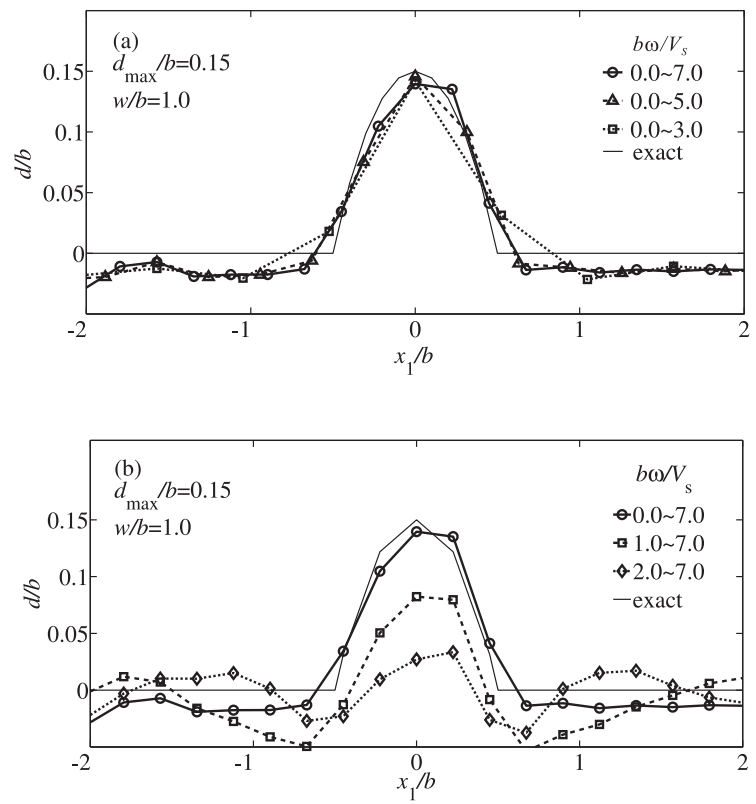

Fig. 6 Reconstructed shapes for sine-curved flaw using 0th symmetric mode, with data in different frequency ranges. (a) Effect of omitting high frequency range data; (b) Effect of omitting high frequency range data.

reconstructed points along $x_{1}$-axis are sparsely located. As a result, the resolution in shape reconstruction becomes less precise. If the frequency range is chosen as $b \omega / V_{\mathrm{s}}=0-3.0$, although the flaw's location is detected, its shape is hard to be recognized from the reconstructed result. Thus, we can say that the high-frequency data controls the accuracy of reconstructed flaw shape.

Next, the effect of low-frequency range on inversion results is considered. Figure 6(b) shows the reconstructed flaw shapes from frequency ranges of $b \omega / V_{\mathrm{s}}=0-7.0 ; 1.0$ 7.0 and 2.0-7.0, respectively. It is observed that, if the low frequency data are missing, the accuracy of the inverse method is much degenerated. Especially, if frequency range
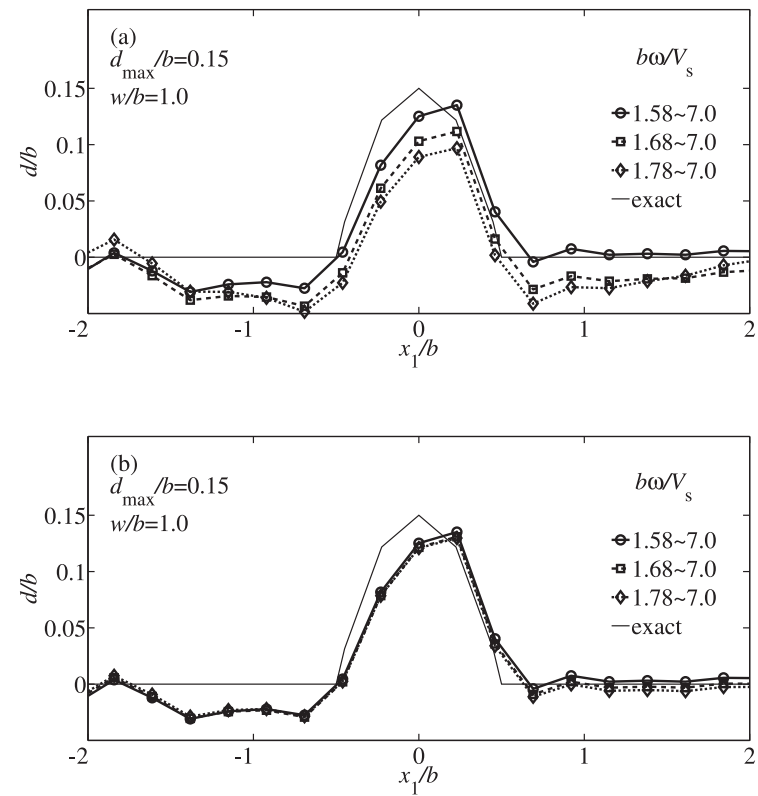

Fig. 7 Reconstructed shapes for sine-curved flaw using 1st anti-symmetric mode, with data in different frequency ranges. (a) Effect of omitting data in near-cutoff frequency range; (b) using extrapolated data in near-cutoff frequency range.

$b \omega / V_{\mathrm{s}}=0.0-2.0$ is absent, the peak of flaw cannot even be distinguished from fluctuations seen on both sides of the flaw. Thus, we conclude that, in our approach, the reflection coefficients in low-frequency range are of vital importance for the detection of flaw.

The sensitivity of frequency range for the 1 st antisymmetric mode $(n=1)$ can also be evaluated. The 1 st anti-symmetric mode has the cutoff frequency at $b \omega / V_{\mathrm{s}}=$ $\pi / 2$. For practical or experimental applications, the reflection coefficients near the cutoff frequency are usually difficult to obtain. We now consider the situation in that the data in the near-cutoff frequency range are not available. Figure 7(a) illustrates the reconstructed results using 1st anti-symmetric 

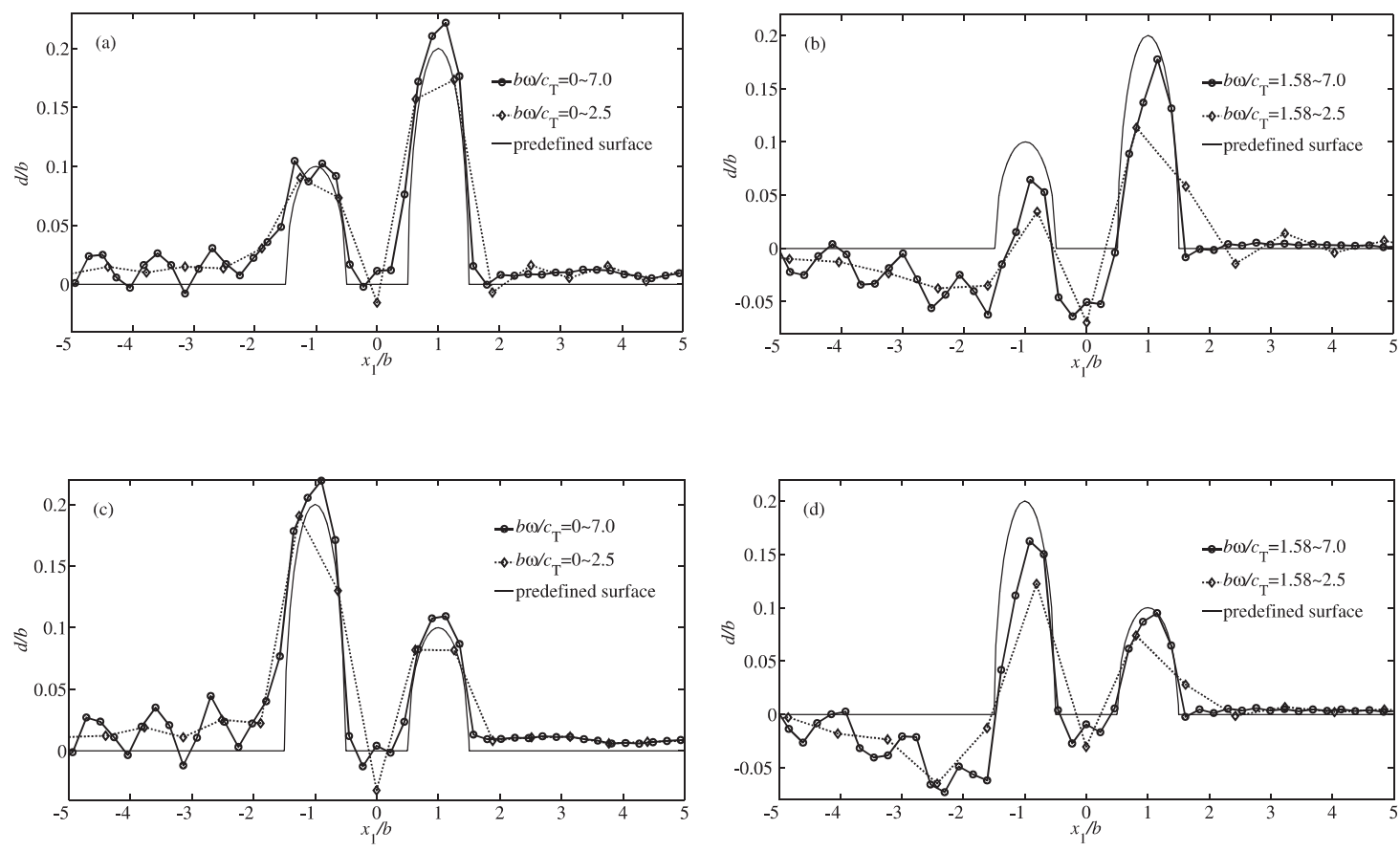

Fig. 8 Reconstructed shapes of double-elliptic-notch flaws. (a) $d_{\max }^{\mathrm{A}} / b=0.20, d_{\max }^{\mathrm{B}} / b=0.10, x_{1}^{\mathrm{A}}=b, x_{1}^{\mathrm{B}}=-b, 0$ th symmetric mode; (b) $d_{\max }^{\mathrm{A}} / b=0.20, d_{\max }^{\mathrm{B}} / b=0.10, x_{1}^{\mathrm{A}}=b, x_{1}^{\mathrm{B}}=-b, 1$ st anti-symmetric mode; (c) $d_{\max }^{\mathrm{A}} / b=0.20, d_{\max }^{\mathrm{B}} / b=0.10, x_{1}^{\mathrm{A}}=-b, x_{1}^{\mathrm{B}}=b$, 0 th symmetric mode; (d) $d_{\text {max }}^{\mathrm{A}} / b=0.20, d_{\text {max }}^{\mathrm{B}} / b=0.10, x_{1}^{\mathrm{A}}=-b, x_{1}^{\mathrm{B}}=b, 1$ st anti-symmetric mode.

mode with different frequency ranges: $b \omega / V_{\mathrm{s}}=1.58-7.0$; $1.68-7.0$ and 1.78-7.0, where in the latter two cases the near-cutoff range is absent. The results show that the data in near-cutoff range play an important role in obtaining good accuracy in flaw reconstruction, since the absence of data in range $b \omega / V_{\mathrm{s}}=1.58-1.78$ lowers the reconstructed depth by as much as $1 / 3$ of $d_{\max }$. However, if we replace the missing data in the range of $b \omega / V_{\mathrm{s}}=1.58-1.78$ with the known reflection coefficient at $b \omega / V_{\mathrm{s}}=1.78$, the result is obviously improved, as shown by Fig. 7(b). Thus, even if the reflection coefficients in near-cutoff range are unavailable, it is better to use the "extrapolated" values from data at higher frequency points than to omit them.

\subsection{Double-notch flaw}

In practical cases, we may encounter the flaw shape with multiple notches. Here an example of two elliptic notches with different depths is considered. The deeper notch A, whose center is located at $x_{1}^{\mathrm{A}}=b$, has the maximum depth $d_{\max }^{\mathrm{A}}=0.2 b$, while the shallower notch $\mathrm{B}$ at $x_{1}^{\mathrm{B}}=-b$ with $d_{\text {max }}^{B}=0.1 b$. Each notch has the width of $w=b$, and the edge distance between two notches is $L=b$, as shown in Fig. 2.

Figures 8(a) and 8(b) show the reconstruction results using the fundamental mode $(n=0)$ and 1 st anti-symmetric mode $(n=1)$, respectively. For each mode, two frequency bands are considered as comparison. It is seen that both modes can reconstruct the depth and width for both notches, while the fundamental mode gives a better accuracy, especially for the cases of smaller frequency bands. Moreover, the deeper notch $\mathrm{A}$ at $x_{1}^{\mathrm{A}}=b$, illuminated by the incident wave, is reconstructed better than the shallower one at $x_{1}^{\mathrm{B}}=-b$ in the shadow zone. Conversely, if the plate is turned around, with $x_{1}^{\mathrm{B}}=b$ and $x_{1}^{\mathrm{A}}=-b$, the shallower notch is reconstructed with higher accuracy, as shown by Figs. 8(c) and 8(d). Thus, for practical usage, it is advisable to send the incident wave from both sides of the flaw area and perform the inversion process twice.

\section{Discussions}

In Sections 4.2-4.5 we illustrated extensive parametric examples to investigate the effectiveness and accuracy of the inverse reconstruction method. The inversion method is suitable for the locating and sizing of thinning flaws with usual shapes, such as sine shape, V-shape and elliptic shape.

Among the guided wave modes, the lower modes show better accuracy. Also, the shallower flaws are reconstructed more precisely than deeper ones. Two reasons for that are considered: Born approximation and shallow-flaw approximation. Firstly, Born approximation is accurate for weak scatterers, but deeper flaws and higher modes have larger and more complex reflection coefficients, which may contradict with "weak scatterer" assumption. Secondly, in deducing eq. (17) to eq. (18), the approximation $\sin \left(2 \beta_{n} X_{2}\right) \approx 2 \beta_{n} X_{2}$ was used, when $\beta_{n} X_{2}$ is a small value. However, for higher modes, $\beta_{n}$ is larger, and as the flaw becomes deeper, $X_{2}$ is increased. As a result, the shallow-flaw approximation is no longer appropriate for deep flaws and high-order modes. We suggest using the 0th symmetric mode or 1 st anti-symmetric mode for the inversion of the flaws with $d_{\max } / b \leq 0.25$ in the application of our inverse method.

The choice of frequency range also directly affects reconstruction accuracy. The data in low frequency range is vital in locating the flaw, while higher frequency data can increase the accuracy of the reconstructed shape. For 1st antisymmetric mode, the reflection coefficients in near-cutoff 
range are important to obtaining the flaw depth. However, they might be difficult to obtain practically. If they are not available, we suggest the extrapolation of values from higher frequency data instead of ignoring them.

We also observed that the part of flaw illuminated by the incident wave is reconstructed better than the shadow zone. Thus, in the case of double notches, it is advisable to perform the inversion from two sides of the flaw area.

\section{Conclusion}

In this paper, a new inverse approach was proposed to reconstruct the location and shape of plate-thinning from reflection coefficients of guided SH-waves. The validity of this method was illustrated by numerical examples. It was found that there is a limitation in our inversion due to some approximations introduced in the formulation. Especially it is noted that our method can be applied to the cases that Born approximation and the shallow-flaw approximation are reasonably adopted.

Based on the present work, the inverse analysis method can be extended to in-plane Lamb wave modes and guided waves in a three dimensional plate with surface flaws. From the practical point of view, furthermore, experimental verification, followed by field applications, will be investigated in future.

\section{REFERENCES}

1) J. L. Rose: J. Pressure Vessel Technol. 124 (2002) 273-282.

2) A. Raghavan and C. E. S. Cesnik: Shock Vib. Dig. 39 (2007) 91-114.
3) J. E. Michaels and T. E. Michaels: Wave Motion 44 (2007) 482-492.

4) P. D. Wilcox: IEEE Trans. Ultrason. Ferroelct. Freq. Contr. 50 (2003) 699-709.

5) J. Rajagopalan, K. Balasubramaniam and C. V. Krishnamurthy: J. Acoust. Soc. Am. 119 (2006) 872-878.

6) H. Nishino, K. Ogura, T. Komatsu and K. Yoshida: Jpn. J. Appl. Phys. 48 (2009) 094501

7) D. P. Jansen and D. A. Hutchins: Ultrasonics 30 (1992) 245-254.

8) D. P. Jansen, D. A. Hutchins and J. T. Mottram: Ultrasonics 32 (1994) 83-89.

9) E. V. Malyarenko and M. K. Hinders: Ultrasonics 39 (2001) 269-281.

10) K. R. Leonard, E. V. Malyarenko and M. K. Hinders: Inverse Problems 18 (2002) 1795-1808.

11) H. Gao, Y. Shi and J. L. Rose: Review of Progress in Quantitative Nondestructive Evaluation, Vol. 24, ed. by D. O. Thompson and D. E. Chimenti, (Am. Inst. Phys., Melville, 2004) pp. 1788-1794.

12) T. R. Hay, R. L. Royer, H. Gao, X. Zhao and J. L. Rose: Smart Mater. Struct. 15 (2006) 946-951.

13) G. R. Liu and K. Y. Lam: Composite Struct. 34 (1996) 251-261.

14) Y. Y. Wang and K. Y. Lam: Composite Struct. 34 (1996) 409-418.

15) G. R. Liu and S. C. Chen: Comput. Methods Appl. Mech. Eng. 190 (2001) 5505-5514.

16) Z. P. Wu, G. R. Liu and X. Han: Eng. Comput. 18 (2002) 116-123.

17) L. R. F. Rose and C. H. Wang: J. Acoust. Soc. Am. 116 (2004) 154171.

18) C. H. Wang and L. R. F. Rose: Review of Progress in Quantitative Nondestructive Evaluation, Vol. 22, ed. by D. O. Thompson and D. E. Chimenti, (Am. Inst. Phys., Melville, 2002) pp. 1615-1622.

19) L. R. F. Rose and C. H. Wang: J. Acoust. Soc. Am. 127 (2010) 754763.

20) B. A. Auld: Acoustic Fields and Waves in Solids, Vol. 2, (John Wiley and Sons, New York, 1973) pp. 155-161.

21) A. Gunawan and S. Hirose: J. Acoust. Soc. Am. 115 (2004) 996-1005.

22) J. E. Gubernatis, E. Domany and J. A. Krumhansl: J. Appl. Phys. 48 (1977) 2804-2811.

23) J. E. Gubernatis, E. Domany, J. A. Krumhansl and M. Huberman: J. Appl. Phys. 48 (1977) 2812-2819. 